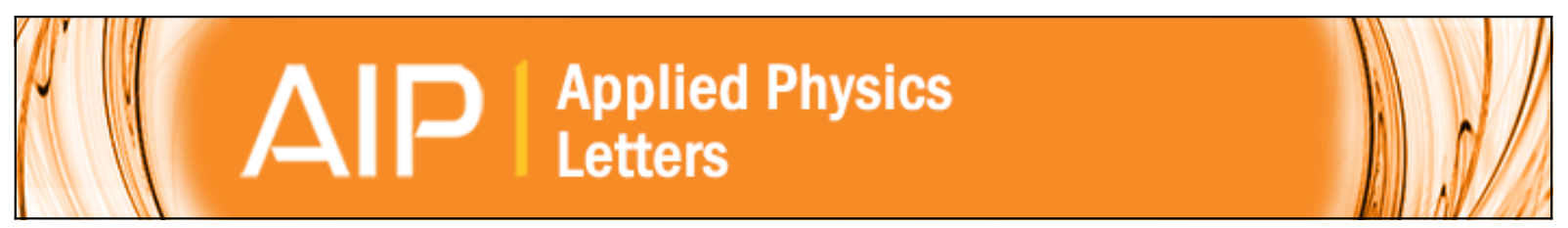

\title{
Local uniaxial tensile strain in germanium of up to $4 \%$ induced by SiGe epitaxial nanostructures
}

Monica Bollani, Daniel Chrastina, Luca Gagliano, Lidia Rossetto, Daniele Scopece, Michael Barget, Valeria

Mondiali, Jacopo Frigerio, Mario Lodari, Fabio Pezzoli, Francesco Montalenti, and Emiliano Bonera

Citation: Applied Physics Letters 107, 083101 (2015); doi: 10.1063/1.4928981

View online: http://dx.doi.org/10.1063/1.4928981

View Table of Contents: http://scitation.aip.org/content/aip/journal/apl/107/8?ver=pdfcov

Published by the AIP Publishing

\section{Articles you may be interested in}

Characterization of locally strained Ge1- x $\mathrm{Sn} x / \mathrm{Ge}$ fine structures by synchrotron X-ray microdiffraction Appl. Phys. Lett. 106, 182104 (2015); 10.1063/1.4921010

The study of temperature dependent strain in Ge epilayer with SiGe/Ge buffer layer on Si substrate with different thickness

Appl. Phys. Lett. 104, 241605 (2014); 10.1063/1.4884063

Room temperature photoluminescence of tensile-strained Ge / Si $0.13 \mathrm{Ge} 0.87$ quantum wells grown on siliconbased germanium virtual substrate

Appl. Phys. Lett. 94, 141902 (2009); 10.1063/1.3114408

Strain-induced ordering of small Ge islands in clusters at the surface of multilayered Si-Ge nanostructures Appl. Phys. Lett. 87, 261919 (2005); 10.1063/1.2151250

Hydrogen gettering and strain-induced platelet nucleation in tensilely strained Si 0.4 Ge 0.6 /Ge for layer exfoliation applications

J. Appl. Phys. 97, 104511 (2005); 10.1063/1.1900928

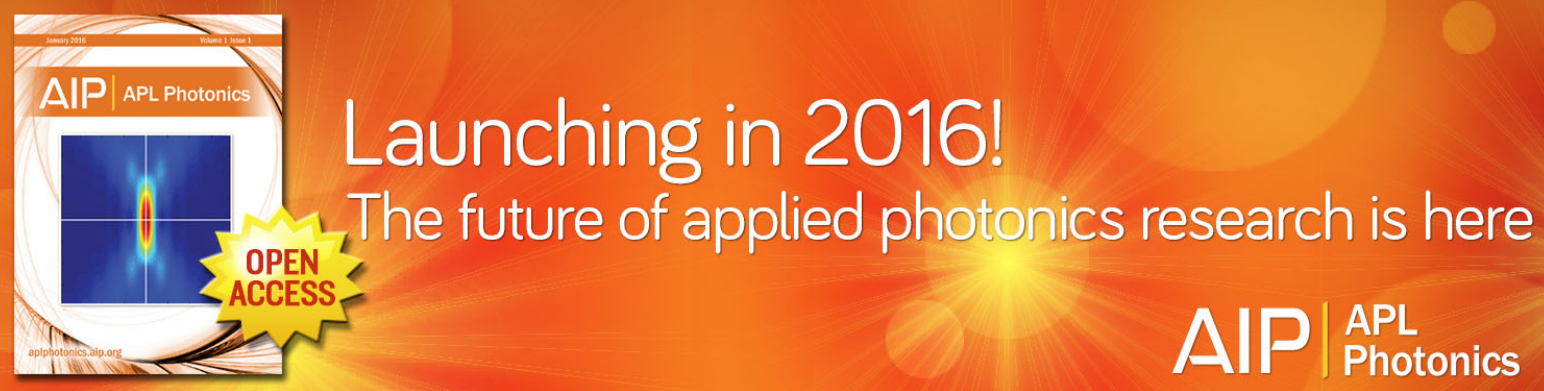




\title{
Local uniaxial tensile strain in germanium of up to $4 \%$ induced by SiGe epitaxial nanostructures
}

\author{
Monica Bollani, ${ }^{1}$ Daniel Chrastina, ${ }^{2}$ Luca Gagliano, ${ }^{3}$ Lidia Rossetto, ${ }^{3}$ Daniele Scopece,, 3 \\ Michael Barget, ${ }^{3}$ Valeria Mondiali, ${ }^{1,2}$ Jacopo Frigerio, ${ }^{2}$ Mario Lodari, ${ }^{2}$ Fabio Pezzoli, ${ }^{3}$ \\ Francesco Montalenti, ${ }^{3}$ and Emiliano Bonera ${ }^{3}$ \\ ${ }^{1}$ IFN-CNR, L-NESS, via Anzani 42, 22100 Como, Italy \\ ${ }^{2}$ L-NESS, Dipartimento di Fisica, Politecnico di Milano, Polo di Como, via Anzani 42, 22100 Como, Italy \\ ${ }^{3}$ L-NESS, Dipartimento di Scienza dei Materiali, Università di Milano-Bicocca, via Cozzi 55 , \\ I-20125 Milano, Italy \\ ${ }^{4}$ Empa - Swiss Federal Laboratories for Materials Science and Technology, Ueberlandstrasse 129, \\ 8600 Dübendorf, Switzerland
}

(Received 30 March 2015; accepted 7 August 2015; published online 25 August 2015)

\begin{abstract}
We show that a relatively simple top-down fabrication can be used to locally deform germanium in order to achieve uniaxial tensile strain of up to $4 \%$. Such high strain values are theoretically predicted to transform germanium from an indirect to a direct gap semiconductor. These values of strain were obtained by control of the perimetral forces exerted by epitaxial SiGe nanostructures acting as stressors. These highly strained regions can be used to control the band structure of silicon-integrated germanium epilayers. (C) 2015 AIP Publishing LLC.
\end{abstract}

[http://dx.doi.org/10.1063/1.4928981]

Germanium is an indirect-gap semiconductor, with the valence band maximum at $\Gamma$ and the conduction band minimum at L. However, a local minimum exists in the conduction band at $\Gamma$ which is only $140 \mathrm{meV}$ above $\mathrm{L}$ at room temperature. ${ }^{1}$ The application of $4 \%-5 \%$ uniaxial strain to $\mathrm{Ge}$, along the $\left[\begin{array}{lll}1 & 0 & 0\end{array}\right]$ direction, is predicted to transform $\mathrm{Ge}$ into a direct-gap semiconductor. ${ }^{2-4}$

A direct-gap semiconductor which is fully compatible with Si-based technology would allow full integration of electronics and optoelectronics and represents a highly sought goal, ${ }^{5,6}$ so various methods of inducing the required strain in Ge micro- and nano-structures are under investigation. ${ }^{7-11}$ Ge microbridges featuring $3 \%$ uniaxial strain along $\left[\begin{array}{lll}1 & 0 & 0\end{array}\right]$ have demonstrated greatly enhanced photoluminescence efficiency, ${ }^{12}$ and even higher strain has been observed in smaller bridges. ${ }^{13}$ In this work, we present an alternative method to induce strain which can be considered significantly easier from the point of view of the fabrication.

It has been shown that patterning of compressively strained SiGe alloys on $\operatorname{Si}\left(\begin{array}{lll}0 & 0 & 1\end{array}\right)$ substrates leads to the transfer of compressive strain into the substrate. ${ }^{14,15}$ Here, we demonstrate the analogous process in which patterned tensile SiGe alloys induce tensile strain in a $\mathrm{Ge}\left(\begin{array}{lll}0 & 0 & 1\end{array}\right)$ substrate. ${ }^{16}$ The actual substrate is a thick $(1 \mu \mathrm{m})$ Ge layer grown on $\mathrm{Si}$ characterized by a complete plastic relaxation. The samples consist of stripes of $\mathrm{SiGe}$ (the stressors) on top of this relaxed Ge layer (the substrate) which are separated by gaps of a few tens of nanometers, Fig. 1(a). Although, in principle, other structures can be used, we chose this geometry because it is the simplest to be fabricated and measured. The partial elastic relaxation of the $\mathrm{SiGe}$ stressors leads to a partial compression of the Ge substrate located under the $\mathrm{SiGe}$ structures, leading to a uniaxially compressed $\mathrm{Ge}$ region (c-Ge). On the other hand, the conservation of the total length of the substrate leads to regions of uniaxially tensile germanium ( $\mathrm{t}-\mathrm{Ge}$ ) in the gaps between the stressors. The geometry of the structures together with the ratio of the lattice parameters and elastic constants of the stressor and the substrate can be used to define the intensity of the perimetral forces marked by arrows on the sketch in panel (a), which are responsible for the strain in the t-Ge. The intensity of these forces can lead to high strain in the gaps, whose magnitude is limited only by the plastic relaxation of elastic energy. ${ }^{16}$

In this work, the Ge content and the geometrical parameters of the stressors (thickness $t$, width $w$, and gap $g$ ) were chosen to maximize the induced strain in the gap taking into account the several compromises analyzed in detail in Ref. 16 , which can be summarized as follows. For what concerns the composition of the stressor, the growth of Ge-rich SiGe allows thicker stressors to be obtained without plastic relaxation, ${ }^{17}$ but results in lower stress forces. For the thickness $t$, a thinner SiGe layer allows a fully strained stressor without
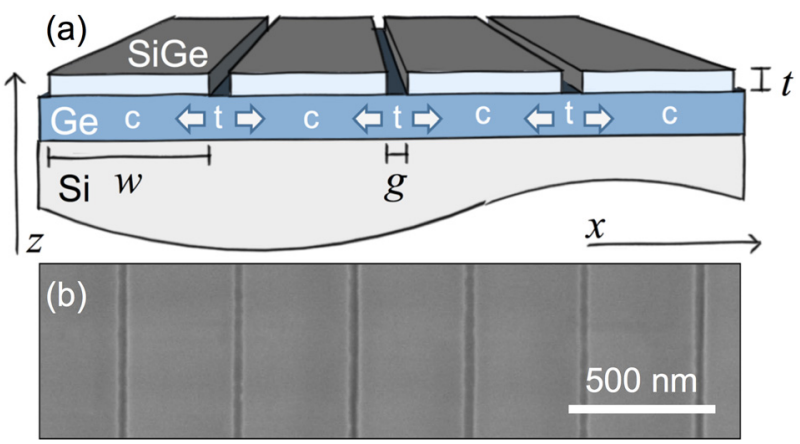

FIG. 1. (a) Sketch of the sample and the reference system (not to scale). SiGe stripes of thickness $t$ and width $w$ are patterned on top of a $1 \mu \mathrm{m}$ thick relaxed Ge layer directly grown on $\mathrm{Si}$. The stripes are separated by a gap $g$. The stripes are parallel to the y direction [ $\left[\begin{array}{lll}1 & 1 & 0\end{array}\right]$. The forces exerted by the stripes pull the Ge within the gaps creating a strong deformation along the $x$ direction, parallel to [1 $\overline{1} 0]$. The vertical direction $z$ is oriented along the crystallographic [llll 001 direction. (b) Top-view scanning electron micrograph of the sample with $g=20 \mathrm{~nm}$. 
TABLE I. Geometrical parameter of the SiGe stressors (gap $g$ and width $w$ ) and average strain $\varepsilon_{x x}$ in the gaps as measured by Raman spectroscopy (from the variation $\Delta \omega$ of the Ge mode at about $300 \mathrm{~cm}^{-1}$ ) and calculated by finite element method (FEM)

\begin{tabular}{lcccc}
\hline \hline $\begin{array}{l}\text { gap } g \\
(\mathrm{~nm})\end{array}$ & $\begin{array}{c}\text { width } w \\
(\mathrm{~nm})\end{array}$ & $\begin{array}{c}\text { shift } \Delta \omega \\
\left(\mathrm{cm}^{-1}\right)\end{array}$ & $\begin{array}{c}\varepsilon_{x x}(\text { Raman }) \\
(\%)\end{array}$ & $\begin{array}{c}\varepsilon_{x x}(\mathrm{FEM}) \\
(\%)\end{array}$ \\
\hline $45 \pm 5$ & $355 \pm 5$ & $-7 \pm 1$ & $3.1 \pm 0.8$ & 2.7 \\
$30 \pm 5$ & $370 \pm 5$ & $-8 \pm 1$ & $3.6 \pm 0.8$ & 3.5 \\
$20 \pm 5$ & $380 \pm 5$ & $-9 \pm 1$ & $4.0 \pm 0.8$ & 4.1 \\
\hline \hline
\end{tabular}

plastic relaxation, while higher thickness means higher stress forces. Very thick stressors are not interesting because the top regions can relax elastically without transferring more strain to the substrate. Considering the gap $g$, smaller gaps induce a higher tensile strain but decrease the volume of the highly strained $\mathrm{Ge}$, which can be a problem for its practical exploitation in a device. For the width parameter $w$, the tensile strain increases with the ratio $w / g .{ }^{14}$ However, when $w$ is significantly larger than $g$, the stressor can be considered infinitely large and there is no appreciable increase of strain. $^{16}$

Following these design guidelines, we fabricated epitaxial SiGe stripes on top of an epitaxial $\mathrm{Ge} / \mathrm{Si}(001)$ virtual substrate with three values of $g$ of 45,30 , and $20 \mathrm{~nm}$, keeping an overall pitch of $400 \mathrm{~nm}$, Table I. The stripes are characterized by a thickness $t=50 \mathrm{~nm}$ and an extension of $25 \mu \mathrm{m}$ along the [110] direction, a length that can be considered infinite as compared to the other geometrical parameters. Figure 1(b) shows a scanning electron micrograph of the stripes with $g=20 \mathrm{~nm}$.

A top-down approach has been used to realize the SiGe nanostressors. Heterostructures consisting of $1 \mu \mathrm{m}$ thick relaxed Ge layers and thin tensile SiGe layers were obtained by low-energy plasma-enhanced chemical vapor deposition. ${ }^{18}$ The threading dislocation density in the Ge layers was reduced by annealing, ${ }^{17}$ a process that induced a small residual thermal biaxial tensile strain between $0.1 \%$ and $0.2 \%$ in the Ge layer on cooling to room temperature. The resulting $\mathrm{Ge} / \mathrm{Si}\left(\begin{array}{lll}0 & 0 & 1\end{array}\right)$ layer forms a virtual substrate (VS) for the subsequent growth of a thin $(50 \mathrm{~nm}) \mathrm{Si}_{0.48} \mathrm{Ge}_{0.52}$ layer (henceforth the SiGe layer). The composition and strain state of both the $\mathrm{Ge}$ and $\mathrm{SiGe}$ layers were measured by highresolution X-ray diffraction (XRD) around the $\left(\begin{array}{lll}0 & 0 & 4\end{array}\right)$ and grazing-incidence (2 2 4) Bragg peaks; the Ge layer was found to be under $0.14 \%$ tensile thermal biaxial strain, and the SiGe layer was almost fully coherent with the Ge VS, with a degree of relaxation of only $5 \%$ corresponding to an in-plane tensile strain of $2.08 \% .{ }^{17}$ The same values of strain for SiGe and Ge can be obtained by Raman spectroscopy ${ }^{19,20}$ with an excitation wavelength $\lambda_{\text {exc }}=532 \mathrm{~nm}$ (Fig. 2(a)), which can probe simultaneously the SiGe layer and the $\mathrm{Ge}$ layer. The probed depth equals half of the penetration depth $d_{p}$ due to self-absorption. For this $\lambda_{\text {exc }}, d_{p} / 2$ is about $50 \mathrm{~nm}$ for SiGe and $10 \mathrm{~nm}$ for Ge. ${ }^{21}$ This spectrum is important not only because it allows to the XRD data to be confirmed but also to rule out the presence of an intermixed layer at the $\mathrm{SiGe} / \mathrm{Ge}$ interface. The fabrication of the SiGe nanostressors is based on a combination of electron beam lithography

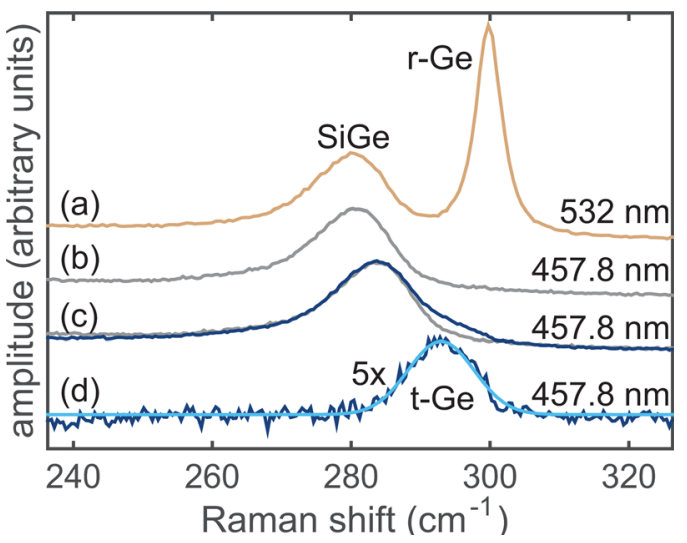

FIG. 2. Raman spectroscopy of the samples before and after patterning. Spectrum (a) was acquired on the sample before patterning with an excitation of $532 \mathrm{~nm}$, enabling the simultaneous measurement of $\mathrm{SiGe}$ and $\mathrm{Ge}$. Spectrum (b) is again taken before patterning, but with a $457.8 \mathrm{~nm}$ excitation, showing that for this wavelength the SiGe layer is opaque and the contribution from Ge below $\mathrm{SiGe}$ is undetectable. The blue spectrum in (c) is a $457.8 \mathrm{~nm}$ measurement of the patterned region (gap $g=45 \mathrm{~nm}$ ) showing the shift to less tensile values of the SiGe band and the appearance of a Gerelated band as a high-wavenumber tail on the SiGe band. In order to highlight the latter, the grey spectrum in (c) is the SiGe band from spectrum (b) shifted to compensate the strain-related shift. Subtracting the grey SiGe spectrum from the blue $\mathrm{SiGe}+\mathrm{Ge}$ spectrum in (c) gives the Ge spectrum in (d) together with its fitting curve.

(EBL) and reactive ion etching (RIE). ${ }^{14,15}$ Scanning electron microscopy (SEM) images and atomic force microscopy (AFM) images have been used to characterize the obtained nanostructures.

Following the reference system described in Fig. 1(a), it is clear that the strongest component of the strain tensor is the $\varepsilon_{x x}$ component, where the $x$ direction is in-plane and perpendicular to the stripes. The finite element method (FEM) simulations in Fig. 3 were obtained with the same settings as in Ref. 16. They show that the surface of the Ge VS under the SiGe stripes is slightly compressed, but that a large tensile strain is induced in the $x$ direction in the Ge VS between the stripes. The effects of the stressors decay rapidly with depth, leaving most of the Ge VS relaxed ( $r-G e)$.

$\mu$ Raman spectroscopy has been used to measure the strain in the $\mathrm{t}-\mathrm{Ge}$ regions, since the Raman shift in $\mathrm{SiGe}$ alloys is dependent on both the Ge content and the overall strain state. ${ }^{19,20,22,23}$ The Ge content dependence allows the signals from the $\mathrm{Ge}$ and $\mathrm{SiGe}$ layers to be easily distinguished. However, the measurement of the strain in the gap is complicated by the small volume of $\mathrm{t}-\mathrm{Ge}$ surrounded by much larger volumes of $\mathrm{r}-\mathrm{Ge}$ and $\mathrm{c}-\mathrm{Ge}$. In order to obtain Raman scattering from only the gap region, a higher excitation energy can be chosen, so that the incoming radiation is absorbed before it reaches the c-Ge underneath the stressors. On the other hand, increasing the excitation energy too far above the Ge Raman resonance (at about $2 \mathrm{eV}$ ) would reduce the intensity of the Ge band. ${ }^{24}$ We observed that an excitation of $\lambda_{\text {exc }}=457.8 \mathrm{~nm}(2.71 \mathrm{eV})$ was high enough to be absorbed completely in the $\mathrm{SiGe}$ layer. For this $\lambda_{\text {exc }}, d_{p} / 2$ for both $\mathrm{SiGe}$ and $\mathrm{Ge}$ is $10 \mathrm{~nm}^{21}$

In Fig. 2(b), one can see that the SiGe band on the flat unpatterned sample is the same as in Fig. 2(a), but the contribution from $\mathrm{Ge}$ is completely removed by the opacity of 

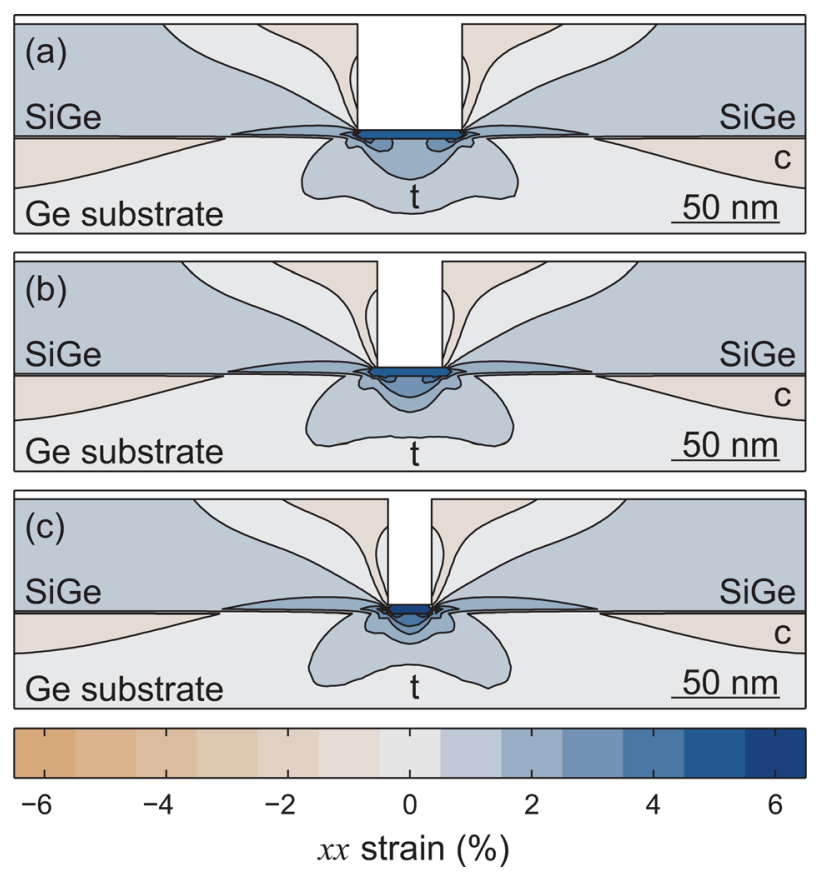

FIG. 3. Simulation by finite-element method of the $\varepsilon_{x x}$ strain component of a system of $\mathrm{SiGe}$ stressors $(x=52 \%)$ on top of a Ge substrate in the region of the high strain. Panels (a), (b), and (c) refer to the sample with gap $g$ equal to 45,30 , and $20 \mathrm{~nm}$, respectively. High tensile strain (marked with t) is induced in the Ge in the gap between the SiGe stressors, within a few nanometers of the surface. The Ge under the stressor is compressed (marked by c).

the SiGe layer for this wavelength. On the other hand, after opening the trenches in SiGe by lithography, we can observe in Fig. 2(c) the appearance of the Ge band as a tail on the high-wavenumber side of the SiGe band, which means that Raman spectroscopy is still efficient enough to observe the tiny amount of Ge not covered by SiGe. Notice that in this spectrum the SiGe band is shifted with respect to spectrum (b), because the SiGe stressor elastically relaxes due to the presence of the trenches. This shift is consistent with the simulations. In order to better highlight the appearance of the Ge band, we superimpose the spectrum to the same spectrum in (b), spectrally shifted in order to compensate for the elastic relaxation of $\mathrm{SiGe}$. The subtraction of two spectra in (c) leads to the Ge contribution, which is shown in spectrum (d) together with a mixed lorentziangaussian fitting.

For all these measurements, the power incident on the sample was of the order of $1 \mathrm{~mW}$. The diffraction limited resolution did not allow the stripes and the gaps to be spatially resolved; the measured spectrum is a superposition of contributions from the $\mathrm{t}-\mathrm{Ge}$ and the surface of the stressor. Thermal artefacts from the heating of the sample were ruled out by observing that the spectral positions of the bands did not change with lower incident powers. The size of the structures is well beyond the critical size for the appearance of effects in the phonon energy due to the quantum confinement. $^{25}$

Figure 4 shows the Ge-Ge phonon band obtained after subtraction of the stressor spectrum. All the spectra were normalized to their maximum intensity. The phonon band moves towards lower energies by almost $10 \mathrm{~cm}^{-1}$, indicating

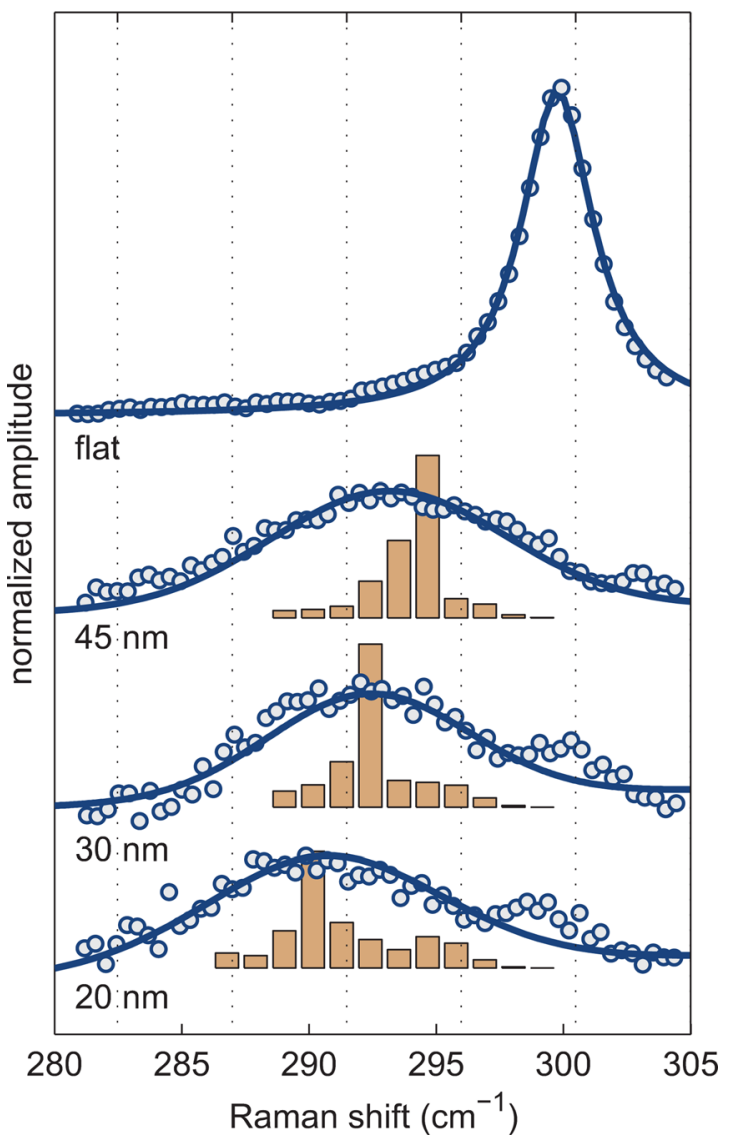

FIG. 4. Raman measurement of strain in Ge. While the lower $x$ scale reports the Raman shift, the upper $x$ scale shows the corresponding value for the $\varepsilon_{x x}$ strain. The upper spectrum refers to a flat, unpatterned sample and is used as a reference. The slightly non-zero strain is the result of the small thermal strain detected also by $\mathrm{x}$-ray diffraction. The lower three spectra result from the measurement of the Ge just below the gap between two adjacent stressors by means of selective resonance Raman spectrocopy in the sample with gap $g=45$, 30, and $20 \mathrm{~nm}$, respectively. The shoulders at $295 \mathrm{~cm}^{-1}$ are an artefact due to stray light. The histogram graphs superimposed to the Raman spectra represent the strain distribution in the tensile Ge as calculated by FEM. The distribution is weighted with an exponential decay mimicking the attenuation of the excitation power within Ge to be directly compared to the Raman spectra.

strong tensile strain. This is a much stronger effect than the $3-4 \mathrm{~cm}^{-1}$ of relative shift observed by Süess et al. in Ref. 12 and the $6-7 \mathrm{~cm}^{-1}$ of relative shift observed by Sukhdeo et al. in Ref. 13.

Since the $\mathrm{t}-\mathrm{Ge}$ is not in a truly biaxial $\left(\sigma_{x x}=\sigma_{y y} ; \sigma_{z z}=0\right)$ or uniaxial $\left(\sigma_{y y}=\sigma_{z z}=0\right)$ state, usual calibrations for strain as a function of relative Raman shift cannot be used. The frequency of a phonon mode depends on the elements of the strain tensor and the phonon deformation potentials according to the dynamical secular equation. ${ }^{26}$ The backscattering geometry from $\left(\begin{array}{lll}0 & 0 & 1\end{array}\right)$ allows only the vertically propagating longitudinal optical mode to be detected. While for some complex systems it is necessary to simulate the spectral response of nanostructures, ${ }^{27,28}$ for the samples presented in this paper, it is possible to translate the Raman shift into a strain with some simplifications justified by the symmetry and the results of the FEM simulations. First, we assume all of the off-diagonal strain components to be zero because for symmetry reasons, and as confirmed by FEM, for most of the sample, the tetragonal symmetry is conserved. It can be 
further assumed that $\varepsilon_{y y}$ is unchanged from the value of $+0.14 \%$ thermal strain as measured by XRD, and that $\sigma_{z z}=0$ since the surface of the $\mathrm{t}-\mathrm{Ge}$ region is free. The values of the normalized deformation potentials are $p=-1.55$ and $q=-2.08$. These values can be retrieved by reversing strain-shift coefficients extracted from experiments and calculations available in the literature and are affected by uncertainties of about $10 \%{ }^{20,29}$ If $b$ and $h$ are the biaxial and hydrostatic strain-shift coefficients, $p$ and $q$ are the result of the following equations:

$$
\begin{aligned}
& p=\frac{1}{\omega_{0}} \frac{2 c_{11}(h-b)}{c_{11}+2 c_{12}}, \\
& q=\frac{1}{\omega_{0}} \frac{2 c_{12} h+c_{11} b}{c_{11}+2 c_{12}},
\end{aligned}
$$

where $c_{11}$ and $c_{12}$ are elements of the elastic stiffness tensor. The calculations yield a strain-shift relation for our experiment of $\Delta \omega\left(\varepsilon_{x x}\right)=-225 \varepsilon_{x x} \mathrm{~cm}^{-1}-0.3 \mathrm{~cm}^{-1}$. Notice that the value of $225 \mathrm{~cm}^{-1}$ is about half of the biaxial strain coefficient, ${ }^{19,23}$ which is consistent with the almost uniaxial nature of the strain. The experimental uncertainty on this figure is of the order of $10 \%$. The $0.3 \mathrm{~cm}^{-1}$ offset takes into account the non-zero value of $\varepsilon_{y y}$.

With this calibration, we can now translate the Raman shift values into strain, and we can notice that the strain of the thinner gap overcomes the $4 \%$ threshold of the indirect to direct transition. In order to compare the results with FEM simulations, we calculate the average strain in the t-Ge region with a width $g$ and a depth equal to $d_{p} / 2$. The values are reported in Table I and are consistent with the experimental results. However, the Raman data and the FEM can be better compared if we consider a weighted average of the strain. The average is weighted with an exponential function which quickly decays with depth (characteristic length $d_{p} / 2$ ) due to the choice of $\lambda_{\text {exc }}$. If we map the strain in the material by binning the strain into intervals of $0.25 \%$, we obtain a distribution that can be readily compared to the experimental spectrum, as shown in Fig. 4. This comparison shows that not only the average of the strain is consistent but also that the width of the spectrum, which is significantly larger than the width of the reference spectrum, can be attributed to the strong strain gradient in the structures which spreads the distribution of the strain and might reduce the phonon lifetime.

Our simulations predict that maintaining a $w / g$ ratio higher than 20 will not continue to significantly increase the strain within the gap. Similarly, a thicker SiGe stressor will not significantly increase the stress in the gap because the additional material on top would be free to relax elastically. The value of $4 \%$ could, in principle, be overcome by further narrowing $g$. However, this can also be a disadvantage because one of the drawbacks of our method is that the volume of $\mathrm{t}-\mathrm{Ge}$ is rather small. With the $g=20 \mathrm{~nm}$ sample, the surface ratio between $\mathrm{t}-\mathrm{Ge}$ and the rest of the Ge is around 1/ 20. Although this value is much higher than the other values in the strained Ge literature, this can be a serious issue if we aim for the realization of an efficient detector. However, it can still be used in all the applications where the injection of the carriers can be controlled spatially such as in an emitter. Also, it must be remembered that in the whole sample the tGe region is expected to be the location of the absolute minimum of energy, and carriers should be attracted and trapped by this region.

In conclusion, we have obtained a controlled tensile strain in a germanium layer by exploiting the forces exerted by epitaxial nanostructures. By means of a simple fabrication method, we use materials and technologies that are fully compatible with the standard silicon technology. This method opens a different path in the realization of strained structures. Raman spectroscopy measurements showed high values of uniaxial tensile strain up to $4 \%$.

We acknowledge the Cariplo Foundation (within the Grant DefCon4 2011-0331) for financial support. One of us (E.B.) would like to thank E. Grilli for support in the spectroscopic measurements.

${ }^{1}$ Landolt-Börnstein: Numerical data and functional relationships in science and technology - New Series, 3rd ed., edited by O. Madelung (SpringerVerlag, New York, 1982), Chap. II, pp. 3-4, 19-22, 43-68, 87-110.

${ }^{2}$ O. Aldaghri, Z. Ikonić, and R. W. Kelsall, J. Appl. Phys. 111, 053106 (2012).

${ }^{3}$ C. Boztug, J. R. Sánchez-Pérez, F. F. Sudradjat, R. Jacobson, D. M. Paskiewicz, M. G. Lagally, and R. Paiella, Small 9, 622 (2013).

${ }^{4}$ G.-E. Chang and H. H. Cheng, J. Phys. D: Appl. Phys. 46, 065103 (2013).

${ }^{5}$ R. Soref, Nat. Photonics 4, 495 (2010).

${ }^{6}$ P. Chaisakul, D. Marris-Morini, J. Frigerio, D. Chrastina, M.-S. Rouifed, S. Cecchi, P. Crozat, G. Isella, and L. Vivien, Nat. Photonics 8, 482 (2014).

${ }^{7}$ P. H. Lim, S. Park, Y. Ishikawa, and K. Wada, Opt. Express 17, 16358 (2009).

${ }^{8}$ A. Ghrib, M. de Kersauson, M. El Kurdi, R. Jakomin, G. Beaudoin, S. Sauvage, G. Fishman, G. Ndong, M. Chaigneau, R. Ossikovski, I. Sagnes, and P. Boucaud, Appl. Phys. Lett. 100, 201104 (2012).

${ }^{9}$ J. Greil, A. Lugstein, C. Zeiner, G. Strasser, and E. Bertagnolli, Nano Lett. 12, 6230 (2012).

${ }^{10}$ S. Huang, W. Lu, C. Li, W. Huang, H. Lai, and S. Chen, Opt. Express 21, 640 (2013).

${ }^{11}$ G. Capellini, C. Reich, S. Guha, Y. Yamamoto, M. Lisker, M. Virgilio, A. Ghrib, M. E. Kurdi, P. Boucaud, B. Tillack, and T. Schroeder, Opt. Express 22, 399 (2014).

${ }^{12}$ M. J. Süess, R. Geiger, R. A. Minamisawa, G. Schiefler, J. Frigerio, D. Chrastina, G. Isella, R. Spolenak, J. Faist, and H. Sigg, Nat. Photonics 7, 466 (2013).

${ }^{13}$ D. S. Sukhdeo, D. Nam, J.-H. Kang, M. L. Brongersma, and K. C. Saraswat, Photonics Res. 2, A8 (2014).

${ }^{14}$ M. Bollani, D. Chrastina, M. Fiocco, V. Mondiali, J. Frigerio, L. Gagliano, and E. Bonera, J. Appl. Phys. 112, 094318 (2012).

${ }^{15}$ E. Bonera, M. Bollani, D. Chrastina, F. Pezzoli, A. Picco, O. G. Schmidt, and D. Terziotti, J. Appl. Phys. 113, 164308 (2013).

${ }^{16}$ D. Scopece, F. Montalenti, M. Bollani, D. Chrastina, and E. Bonera, Semicond. Sci. Technol. 29, 095012 (2014).

${ }^{17}$ J. Frigerio, M. Lodari, D. Chrastina, V. Mondiali, G. Isella, and M. Bollani, J. Appl. Phys. 116, 113507 (2014).

${ }^{18}$ G. Isella, D. Chrastina, B. Rössner, T. Hackbarth, H.-J. Herzog, U. König, and H. von Känel, Solid-State Electron. 48, 1317 (2004).

${ }^{19}$ F. Pezzoli, E. Bonera, E. Grilli, M. Guzzi, S. Sanguinetti, D. Chrastina, G. Isella, H. von Känel, E. Wintersberger, J. Stangl, and G. Bauer, J. Appl. Phys. 103, 093521 (2008).

${ }^{20}$ F. Pezzoli, E. Bonera, E. Grilli, M. Guzzi, S. Sanguinetti, D. Chrastina, G. Isella, H. von Känel, E. Wintersberger, J. Stangl, and G. Bauer, Mater. Sci. Semicond. Process. 11, 279 (2008).

${ }^{21}$ M. Bollani, D. Chrastina, A. Fedorov, R. Sordan, A. Picco, and E. Bonera, Nanotechnology 21, 475302 (2010).

${ }^{22}$ J. C. Tsang, P. M. Mooney, F. Dacol, and J. O. Chu, J. Appl. Phys. 75, 8098 (1994). 
${ }^{23}$ P. H. Tan, K. Brunner, D. Bougeard, and G. Abstreiter, Phys. Rev. B 68, 125302 (2003).

${ }^{24}$ A. Picco, E. Bonera, E. Grilli, M. Guzzi, M. Giarola, G. Mariotto, D. Chrastina, and G. Isella, Phys. Rev. B 82, 115317 (2010).

${ }^{25}$ I. Campbell and P. Fauchet, Solid State Commun. 58, 739 (1986).

${ }^{26}$ E. Anastassakis, J. Appl. Phys. 81, 3046 (1997).
${ }^{27}$ E. Bonera, F. Pezzoli, A. Picco, G. Vastola, M. Stoffel, E. Grilli, M. Guzzi, A. Rastelli, O. G. Schmidt, and L. Miglio, Phys. Rev. B 79, 075321 (2009).

${ }^{28}$ R. Singh, E. J. Dailey, J. Drucker, and J. Menéndez, J. Appl. Phys. 110, 124305 (2011).

${ }^{29}$ Z. Sui, H. H. Burke, and I. P. Herman, Phys. Rev. B 48, 2162 (1993). 